\title{
A Noise-Robust Image Encryption Algorithm Based on Hyper Chaotic Cellular Neural Network
}

Gangyi Hu, Jian Rong, Weili Kou

College of Big Data and Intelligence Engineering, Southwest Forestry University, China hugangyi604@126.com

swordrong@163.com

kwl_eric@163.com

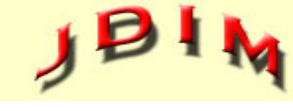

Journal of Digital Information Management

Sumeth Yuenyong

Department of Computer Engineering, Faculty of Engineering, Mahidol University, Thailand sumeth.yue@mahidol.ac.th

Jian Qu

Department of Engineering Technology, Panyapiwat Institute of Management, Bangkok, Thailand lordjohnquest@gmail.com

ABSTRACT: We propose an image encryption algorithm based on a 6-dimensional chaotic cellular neural network $(C N N)$ that is robust to noise/missing pixels in the cipher image. We performed parameter search on the templates of the CNN in order to discover the parameters that leads to $6 D$ chaotic evolution of the state, and then used the resulting chaotic sequence as the basis of encryption. The encryption process itself consists of shuffling the positions of image pixels based on the numerical value of the chaotic sequence; the second half of the encryption process consists of changing the shuffled image pixel values by performing XOR operation between the pixel values and the numerical value of the chaotic sequence. By using simple operations like sorting and XOR in the encryption process, the algorithm is robust to noise/ missing pixels in the cipher image. We illustrate this by comparing the robustness against 3 recently proposed chaos-based image encryption algorithms. The results show that our algorithm is competitive with the state-ofthe-art in term of encryption security, and superior in term of robustness.

Subject Categories and Descriptors

I.4 [Image Processing and Computer Vision]; E.3 [Data Encryption]; F1.1 [Models of Computation neural networks]

General Terms: Neural Networks, Image Encryption
Key Words: Cellular Neural Networks, Hyper Chaotic System, Image Encryption, Noise-Robust

Received: 16 March 2018, Revised 2 May 2018, Accepted 13 May 2018

DOI: $10.6025 / \mathrm{jdim} / 2018 / 16 / 5 / 246-257$

\section{Introduction}

Encryption of images using chaos is an active field of research. However, the focus is on improving the encryption security according to some measure such as correlation between adjacent pixels or information entropy[1,5,23,27]. Other desirable aspects of encryption such as robustness have not received any attention, even by very recent works[10,15]. In this paper, we propose a chaos-based encryption algorithm that is robust to additive noise and missing pixels in the cipher image. This is a highly desirable property in situations where sensitive images are being sent through a wireless RF channel that is subjected to noise or interruptions that may cause the transmission to be incomplete; for example, aerial surveillance drones operating in challenging environments like bad weather, conflict zones, etc.

The cellular neural networks (CNN) have the characteristics of parallel processing and chaos, it has a regular structure, 
and each cell unit is coupled to a neighboring cell. Hence, it is easy to realize as very large-scale integration (VLSI). Higher order dimensional CNN is more capable for producing super chaotic behavior. The 6- dimensional chaotic cellular neural network (6D CNN) has gradually become a hot research topic in image encryption because of it high dimensional and perfect chaos performance. This proposed algorithm is based on a 6-dimensional chaotic cellular neural network (6D CNN). The encryption key is the initial state of the network plus the step size parameter to make the discrete-time state transitions. The $6 \mathrm{D}$ chaotic sequence produced is used as the basis of encryption. A number of experiments was conducted to verify the effectiveness of the proposed scheme. Specifically by adding noise to and destroying parts of the cipher images, the results show that the proposed method have superior robustness to noisy and damaged cipher images than recent chaos-based encryption algorithms.

\section{Background and Related Works}

\subsection{Image Encryption}

Image encryption is the process of hiding the content of an image from unintended viewer by making it looks like noise. Perhaps the earliest well-known work in this field was[14], in which an image was passed through logical operations with a chaotic sequence generated by a key. The sequence used was only $1 \mathrm{D}$ and hence the security was low. The next major work was[7], where the cat map was used to shuffle an image. A few years later [18] proposed an algorithm based on the logistic map, which is still used as a subroutine in many more recent works. Chaos-based encryption was used again in [13], and it was then followed by [12], which is most similar to our work. The main difference is that our work uses higher dimensional chaos and the chaos sequence is used directly in the pixel permutation process, instead of the logistic map. From around 2005 most works on image encryption use chaos in one way or another as the basis of encryption; the difference among them being the source of chaos[28,30], combining different chaotic sequences[2] or introducing more parameters in the generation of the chaos sequence in order to increase the security [26]. More recent works combined chaos with other approaches in order to improve the encryption effectiveness such as bit-level permutation [31] on or by borrowing ideas from physics like diffusion[11]. However, this mean more complicated algorithm and less robustness since the approach being combined with chaos may be sensitive to noise in the cipher image, as will be discussed in the next section.

\subsection{Chaos}

Chaos is a behavior of deterministic nonlinear systems under certain conditions [22]. The state evolution of a chaotic system is extremely sensitive to the initial state and the system parameters. For the same chaotic systems with exactly the same parameters and a very small difference in the initial state, the long term evolution of the system state will be very different, making the long term behavior of a chaotic system unpredictable. This unpredictability of the state sequence is what makes it useful for encryption; without the key, it is virtually impossible to predict; but if the key is available it can be generated exactly on the receiving end of an encrypted communication to decrypt the message. Chaos also forms the basis of many encryption schemes that relies on it to provide the element of randomness [20,21,24], where chaos is used in conjunction with some mathematical operation/procedure to achieve better encryption security. This approach is not good from the noiserobustness perspective, because the added procedure may be more sensitive to noise. For example, in [19] there is a feedback path that goes back to the front of the pixel substitution and permutation process. If there is noise involved, it will enter this feedback loop and increasingly corrupt the process as more iterations are performed. Or in [3], where some significant pixels (defined in their paper) which are essential to the decryption process may be missing altogether from corruption in the cipher image, leading to incorrect decryption. For this reason, in this work we focus on the old-style direct approach [14] where the chaos sequence is used directly as the basis of encryption and aim to achieve high security from simply the high dimensionality of the chaotic sequence, together with operations that are performed on the chaotic sequence such that they can be replicated exactly at the receiver regardless of corruption in the cipher image. A basic flowchart of image encryption and decryption using chaos is shown in Figure 1.

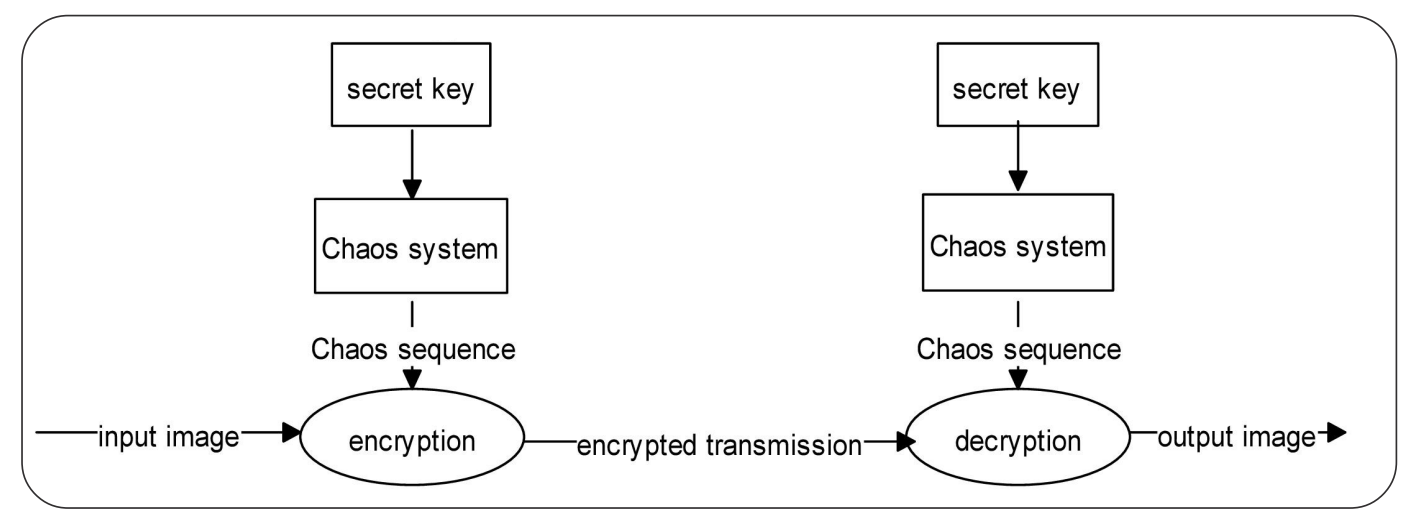

Figure 1. Encryption and decryption using chaotic sequence 
The source of the chaotic sequence can be any nonlinear system but we choose CNN because it can give arbitrary high dimensional chaos (as long as suitable parameters can be found).

\subsection{Cellular Neural Network}

CNN was proposed by [9]. Its basic unit are cells, which are arranged in a planar 2- D lattice. CNN's unique characteristic that sets it apart from other types of neural network is local connectivity; each cell only have connections to cells within its neighborhood. Denoting the cell at row $i$ and column $j$ as $C_{i j}$, its neighborhood can be defined as

$N_{i j}(r)=\left\{C_{a b} \max (|a-i|,|b-j|) \leq r, 1 \leq a \leq M, 1 \leq b \leq N\right\}$

Where $1 \leq i \leq M, 1 \leq j \leq N, r$ is the radius of the neighborhood of cell $C_{i j}$, and $C_{a b}$ is the neighbor cell of cell $C_{i j}$.

A cell is composed of a circuit which can be modeled by the first order nonlinear differential equation.

$$
C \frac{d x_{i j}(t)}{d t}=-\frac{X_{i j}(t)}{R_{x}}+\sum_{k, l \in N_{i j}(r)} A_{k l} y_{k l}(t)+\sum_{k, l \in N_{i j}(r)} B_{k l} u_{k l}+I_{i j}
$$

Where $X_{i j}$ is a state variable, $Y_{k 1}$ is the outputs of cells, $U_{k 1}$ is the input of cells, $C$ and $R_{x}$ are system constants, $I_{i j}$ is the threshold, $A$ is the feedback parameter matrix and $B$ is the control parameter matrix. The subscripts after the matrices in the equation denote the matrix elements. The behavior of $C N N$ is defined by these parameter matrices. Finally,the output equation of $C N N$ is given by

$$
y_{i j}(t)=\frac{1}{2}\left(\left|x_{i j}(t)+1\right|-\left|x_{i j}(t)-1\right|\right)=f(x)
$$

In order to get chaotic sequences to be used for encryption, we utilized a 6- units $C N N$. Since this is a small size, the neighborhood was defined to be the entire network. The challenge was to discover the proper values for the parameter matrices $A, B$ and $I$ that give rise to chaotic state evolution. In order to get these values, we set the system constants to $C=1$ and $R_{x}=1$, then performed a grid-based parameter search. One parameter set that we discovered that give rise to chaotic state evolution is shown in (4).

$$
\begin{gathered}
\text { A = 0 except } a_{44}=404 ; \mathbf{I}=\mathbf{0} ; \\
B=\left[\begin{array}{cccccc}
0 & 0 & -1 & -1.2 & 0 & 0 \\
0 & 2 & 1 & 0 & 0 & 0 \\
11 & -12 & 0 & 0 & 0 & 0 \\
92 & 0 & 0 & -95 & 1 & -1 \\
0 & 0 & 5 & 0 & -1 & 0 \\
0 & 0 & 0 & 5 & 0 & -12
\end{array}\right]
\end{gathered}
$$

Substituting (3) and (4) into (2) and simplifying, we obtained the following state evolution equations for each of the 6 cells in the network. Note that we dropped the second subscript and simply use a single subscript to denote the different cells of the network.

$$
\left\{\begin{array}{l}
\frac{d x_{1}}{d t}=-x_{3}-1.2 * x_{4} \\
\frac{d x_{2}}{d t}=2 * x_{2}+x_{3} \\
\frac{d x_{3}}{d t}=11 * x_{1}-12 * x_{2} \\
\frac{d x_{4}}{d t}=92 * x_{1}-95 * x_{4}+x_{5}-x_{6}+202 * \\
\frac{d x_{5}}{d t}=5 * x_{3}-x_{5} \\
\left.\frac{d x_{6}}{d t}=5 * x_{4}-12 * x_{6}+1|-| x_{4}-1 \mid\right)
\end{array}\right.
$$

Using(5), we can generate the $6 \mathrm{D}$ chaotic sequence that forms the basis of encryption. The step-size parameter $h$ can be chosen freely to a small value, which we set at 0.005 . The initial value of $x_{i}$ (where $i=1,2 \ldots 6$ ) can be set to arbitrary values, each with any number of digits (up to machine precision). The initial state is the seed that starts the generation of chaotic sequence from the evolution of $x_{i}$, hence, it is the key for the encryption. As long as the parameters given in (4) is used, any key would give rise to chaotic evolution of the CNN state. As an example, when the initial state is set as $x_{1}(0)=0.1, x_{2}(0)=x_{3}(0)=x_{4}$ $(0)=x_{5}(0)=x_{6}(0)=0.2$; the $\mathrm{CNN}$ generates chaotic attractors as shown in Figure 2. It will be demonstrated later in the paper that the encryption is highly sensitive to the key. That is, during decryption, if the key is even slightly wrong, the cipher image cannot be correctly recovered.

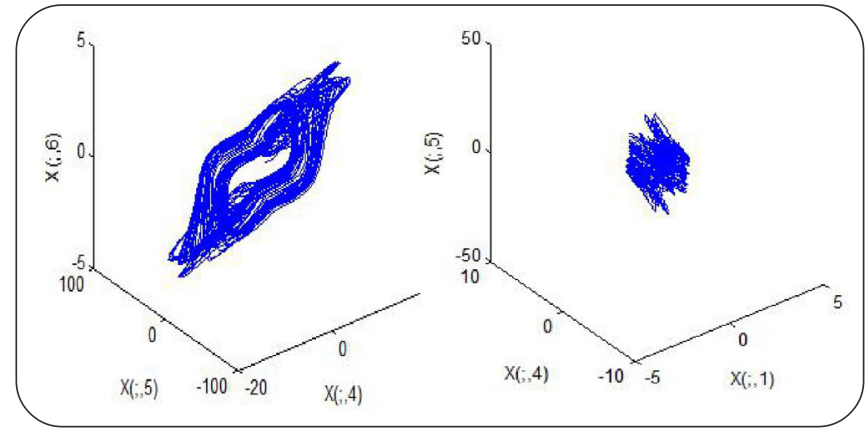

Figure 2. The chaotic attractors generated by the 6D CNN from the key

Using chaos sequences generated from $\mathrm{CNN}$ for encryption had been considered in the literature. Some recent results includes $[4,8,17]$. Bigdeli et.al. proposed a hybrid architecture based on CNN they called chaotic neuron layer and permutation neuron layer. Their focus was on the performance for color images[14]. Chen et. al. proposed using dynamic key for CNN where the key is derived from the image to encrypted itself. This leads to 
high encryption security as can be measured by information entropy, but the cipher key is vulnerable to noise; even a small amount would prevent correct recovery of the original image from the corrupted cipher image [8]. Lin et. al. used CNN along with Latin squares, the problem with this approach is that the large Latin square itself is part of the encryption process and hence part of the key, which requires a lot of numbers $\left(256^{2}\right)$ to represent, in contrast, the key consists of only 7 numbers in our approach.

To conclude the literature survey, we found that chaos had been used extensively in image encryption, and recently the trend was to combine chaos with other approaches in order to increase the encryption security. This does come with the cost of robustness, as will be shown in the experiments section. In the next section we introduce our algorithm.

\section{Methods}

Our image encryption process using $6 \mathrm{D}$ chaotic $\mathrm{CNN}$ may be summarized as follows:

1. Choose the initial state and step size that makes up the key.

2.Update the CNN state using (5) a large number of times to get sufficiently long chaotic sequence, e.g., the same length as the number of pixel in the image to be encrypted.

\section{Shuffle the pixel positions.}

4.Change the pixel values of the pixel-shuffled image from the previous step.

The output of this step is the cipher image.

Where the basis for steps 3 and 4 above is the $6 \mathrm{D}$ chaotic sequence obtained from the $\mathrm{CNN}$. We represent this sequence by a $6 \times L$ matrix $S$, where $L$ is the number pixels in the image to be encrypted and each column of $S$ is a dimension of the chaotic sequence. The pixel shuffling process is given in Algorithm 1. The basic idea of Algorithm 1 is to shuffle the image according to the permutation order that comes from sorting each dimension of the 6D chaotic sequence numerically.

After the pixel positions are shuffled, the next step (step 4 above) is to change the intensity value of each pixel in each channel. We utilized the XOR operation for this purpose. The process is given in Algorithm 2, in which the 6D chaotic sequence matrix $S$ is transformed by taking linear combination between column $i$ and column $i+3$. The purpose of multiplying with integers $N_{1}$ and $N_{2}$ is to scale up the value of the chaotic sequence to be in the scale of 1000 , in order to go through the mod operation with 256. After the chaotic sequence had been transformed, the XOR operation is performed between each pixel of $P$ (the pixel-shuffled image) and each element of the transformed chaotic sequence in order to obtained the final cipher image $C$.

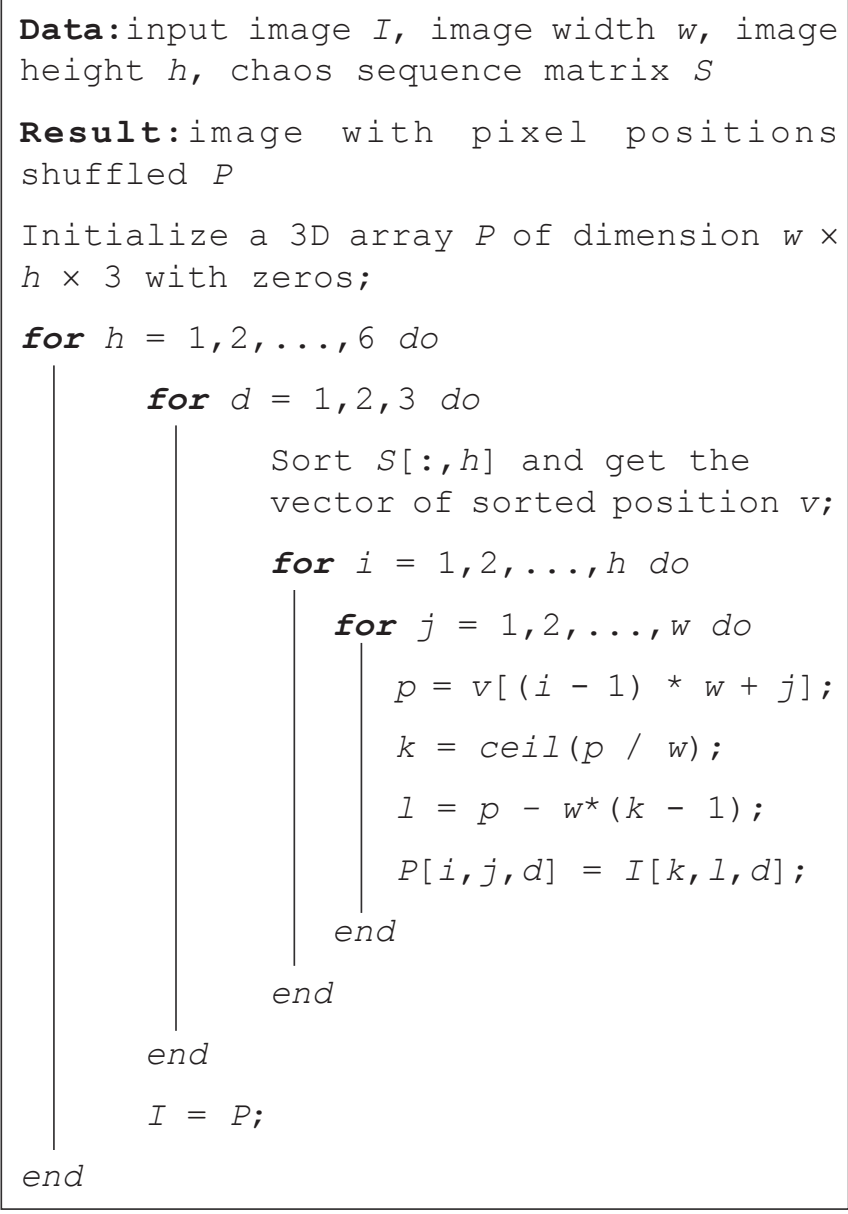

Algorithm 1. Pixel shuffling procedure

The decryption process is just the reverse of the encryption. The receiver use the key to generate the same chaotic sequence, run Algorithm 2 again to get the pixel intensities back, the use the sorted position vector $v$ in Algorithm 1 to recover the pixel positions.

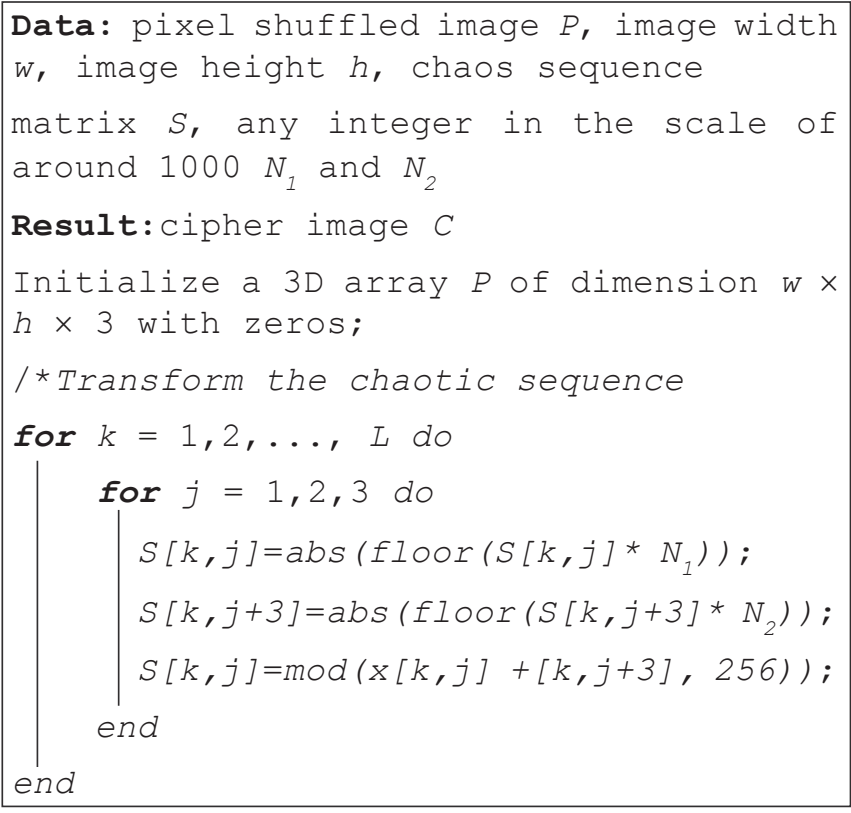




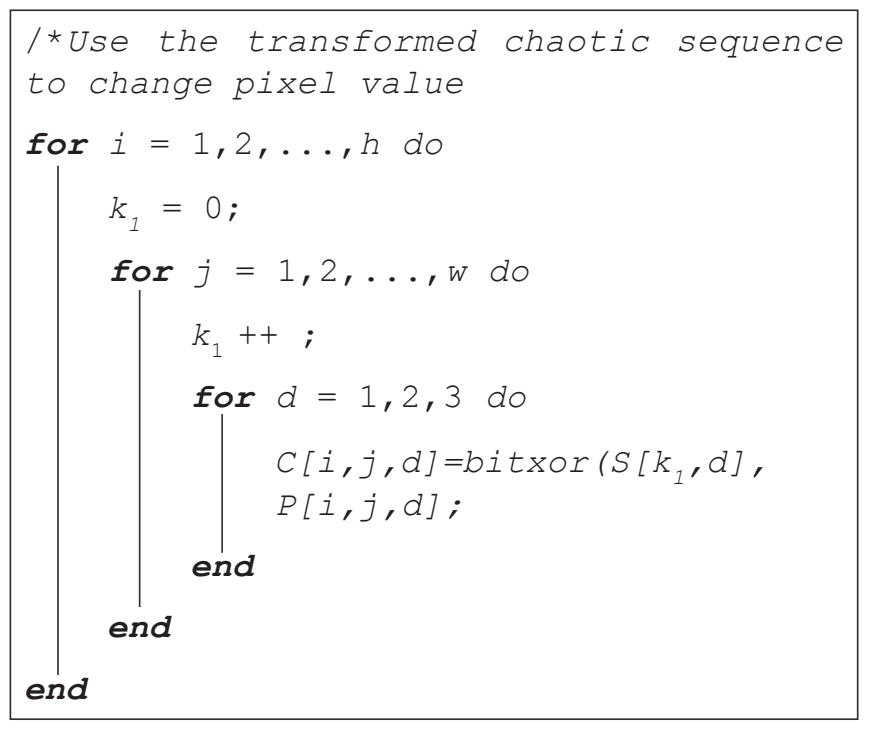

Algorithm 2. Pixel intensity changing procedure

Since our approach consists of sorting and the XOR operation, noise/missing pixels in the cipher image does not affect the shuffling operation because the receiver with the key can generate exactly the sequence that was used to perform shuffling and un-shuffle the noisy/damaged cipher image back exactly. Noise/missing pixels (a missing pixel can be thought of as containing so much noise that it exceed the maximum pixel value and become white) then affects only the gray-value changing operation. Consider the truth table of the XOR operation as shown in Figure 3. Let $X$ represent a bit of a pixel value and $Y$ represent the a bit of a value in the chaotic sequence.
Since $Y$ can be regenerated exactly at the receiver, its value will not change. The only possible error in the output $Z$ then comes from bit-flipping of $X$, which will also cause the output $Z$ to flip. However, because the XOR operation is performed bit-wise, bit-flipping on any bit does not affect any other. This is the source of our algorithm's noise robustness.

\begin{tabular}{|l|l|c|}
\hline \multicolumn{2}{|c|}{ Inputs } & Outputs \\
\hline $\mathrm{X}$ & $\mathrm{Y}$ & $\mathrm{Z}$ \\
\hline 0 & 0 & 0 \\
\hline 0 & 1 & 1 \\
\hline 1 & 0 & 1 \\
\hline 1 & 1 & 0 \\
\hline
\end{tabular}

Figure 3. The truth table of the $X O R$ operation

\section{Results and Discussion}

\subsection{Demonstration for the Effectiveness of the Proposed Algorithm}

In the first round of experiments we performed basic check of the proposed algorithm's effectiveness. We used the well-known Lena and Cameraman images with size $256 \times$ 256. The encryption results are showed in Figure 4, $(a, e)$ are the original images, $(b, f)$ are the histograms of the original images, $(c, g)$ are the cipher images, and $(d, h)$ are the histograms of the cipher images, it can be seen that the distribution of the cipher images are uniform and the cipher images look completely random.

\begin{tabular}{|l|l|l|l|l|}
\hline & $\begin{array}{l}\text { Lena } \\
\text { Original Image }\end{array}$ & $\begin{array}{l}\text { Lena } \\
\text { Cipher Image }\end{array}$ & $\begin{array}{l}\text { Cameraman } \\
\text { Original Image }\end{array}$ & $\begin{array}{l}\text { Cameraman } \\
\text { Cipher Image }\end{array}$ \\
\hline Horizontal direction & 0.9606 & 0.0298 & 0.9778 & 0.0074 \\
\hline Vertical direction & 0.9499 & 0.0523 & 0.9793 & 0.0052 \\
\hline Diagonal direction & 0.9585 & 0.0150 & 0.9686 & 0.0089 \\
\hline
\end{tabular}

Table 1. The Correlation Coefficient from the Three Directions of the image

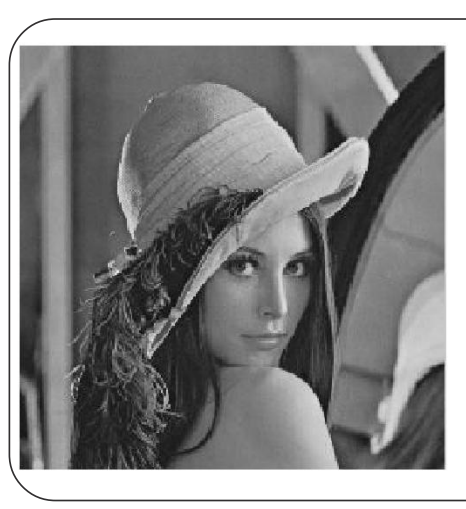

(a) The lena original image

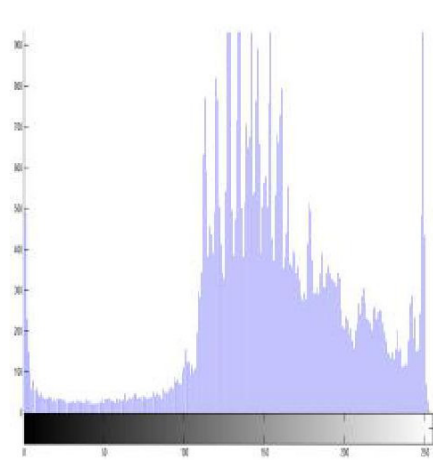

(b) The histogram of original image

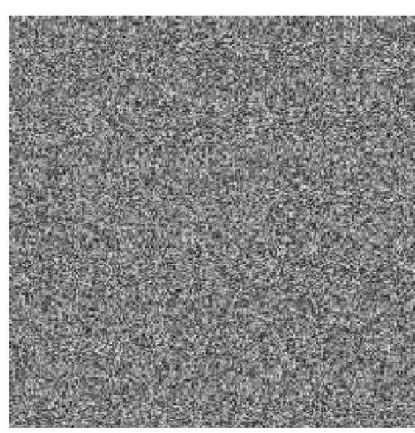

(c) The cipher image image 


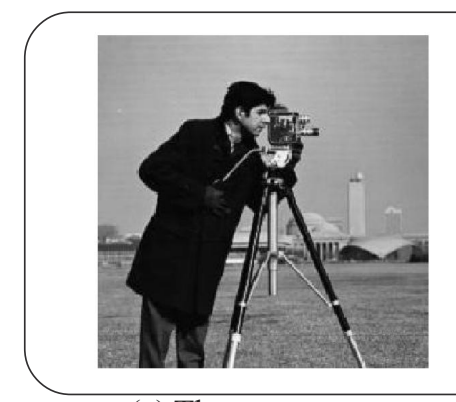

(a) The cameraman original image

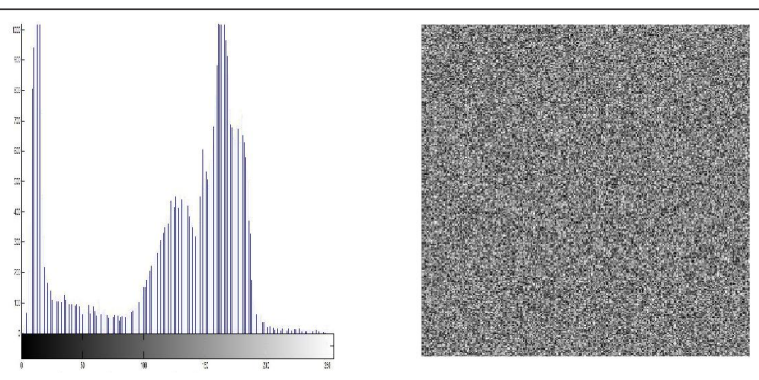

$\begin{array}{ll}\text { (a) The cameraman } & \text { (c) The cipher image }\end{array}$ original image

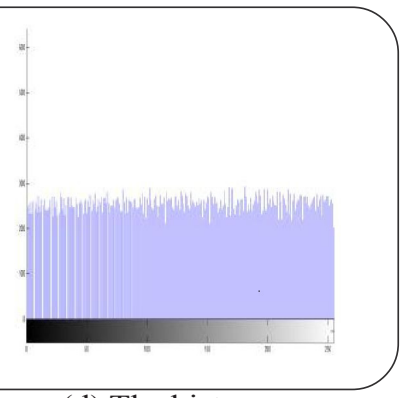

(d) The histogram of cipher image

Figure 4. The encryption results of the Lena and Cameraman images

\subsubsection{Correlation Analysis of Adjacent Pixels}

We randomly selected 1000 pairs of adjacent pixels in the original image Lena and its cipher image, measured the correlation coefficients according to (6) in three different directions: horizontal, vertical and diagonal.

$$
r_{x y}=\frac{E(x y)-E(x) E(y)}{\sqrt{D(x)} \sqrt{D(y)}}
$$

Where $E$ is the expectation operator and $D$ is the variance, the correlation of the adjacent pixel test results for the Lena and Cameraman image encryption are shown in Table I, and the correlation effect of adjacent pixel in horizontal direction is shown in Figure 5. It can be seen that there is

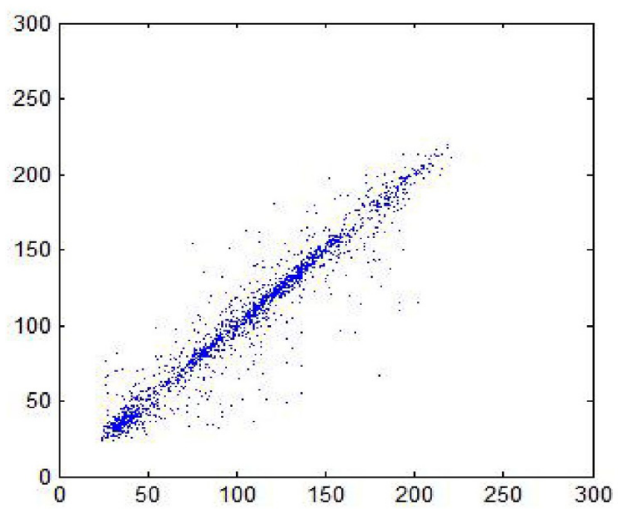

(a)The pixel position of Lena original image



(c)The pixel position of Cameraman original image high correlation between adjacent pixels in the original image,after encryption,the correlation value between adjacent pixels of the cipher image is very low.

\subsubsection{Analysis of the NPCR}

The number of pixel change ratio (NPCR) is defined by (7). If its value is high, changing only a single pixel in the original image will lead to a very different cipher image.

$$
\begin{aligned}
& N P C R=\frac{\sum_{i=1}^{M} \sum_{j=1}^{N} R(i, j)}{M^{*} N} * 100 \%, \\
& R(i, j)= \begin{cases}1, & c_{1}(i, j) \neq c_{2}(i, j) \\
0, & c_{1}(i, j)=c_{2}(i, j)\end{cases}
\end{aligned}
$$

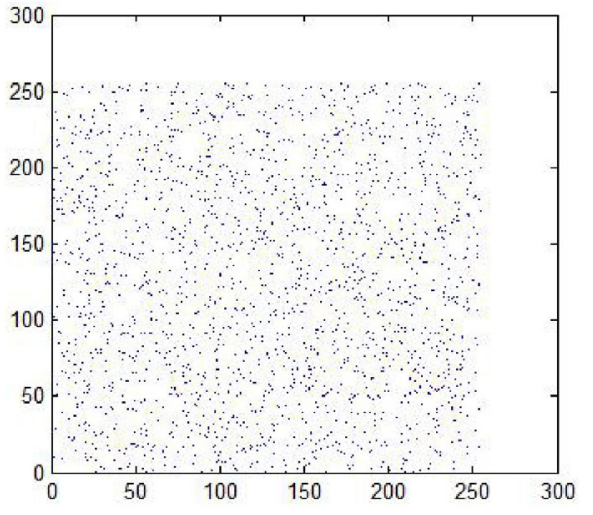

(b)The pixel position of Lena cipher image

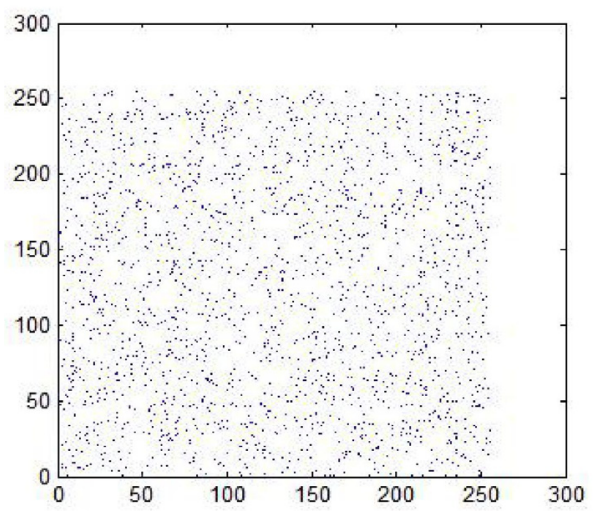

(d)The pixel position of Cameraman cipher image

Figure 5. The correlation analysis of the adjacent pixel 
where $M$ and $N$ represents the image length and width in pixels, respectively. $c_{1}$ and $c_{2}$ represents the two cipher images that had only one pixel with different value in the original image. $c_{1}(i, j)$ and $c_{2}(i, j)$ respectively represent the row $i$ and column $j$ pixel value of $c_{1}$ and $c_{2}$.

In the simulation experiment, when one image pixel value of the Lena is changed, according to (7), the value of NPCR is 0.9960 , it shows that the sensitivity of this algorithm to pixel change in the original image is very high.

\subsubsection{Analysis of the Image Information Entropy(IE)}

The image information entropy reflects the gray distribution of the image, if the image pixel value distribution is perfectly uniform, it means that the information entropy is the largest, and the cipher image is the most random. The information entropy of the Lena original image and Lena cipher image are 7.3897 and 7.9913 , respectively. It can be seen that the image information entropy of the cipher image is close to the maximum information entropy value of eight (because the image is 8-bits) for gray images.

\subsubsection{Application to Color Image}

In order to verify the encryption and decryption effectiveness of this algorithm for color images, we randomly select an image of dinosaur, the encryption and decryption results are shown in Figure 6.

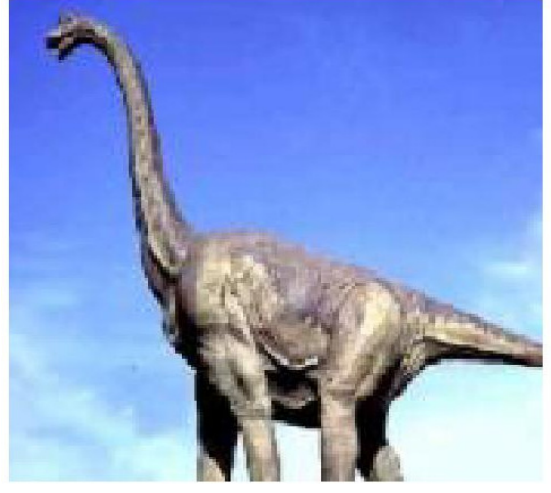

(a)The original image

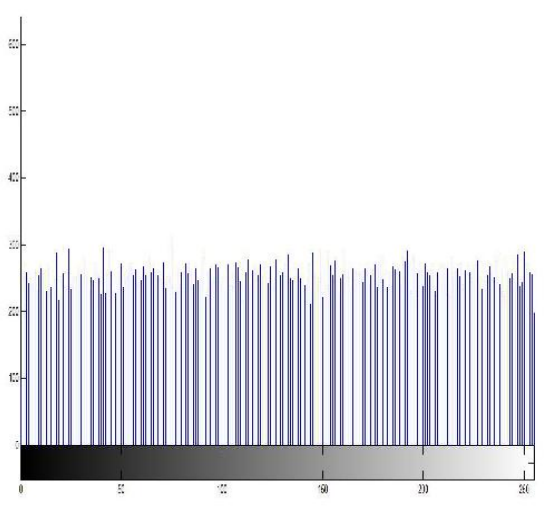

(d) The histogram of cipher image

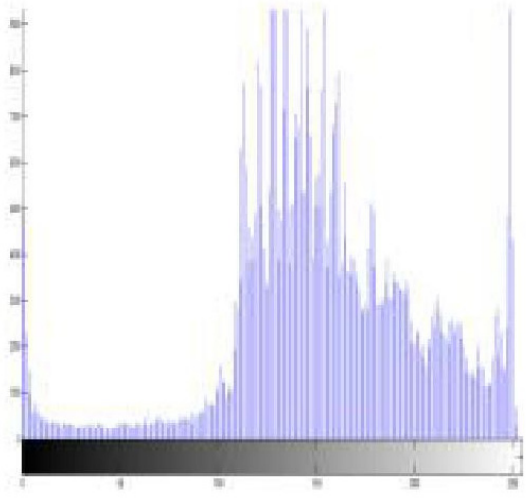

(b)The histogram of original image



(e) Decrypted image using correct key

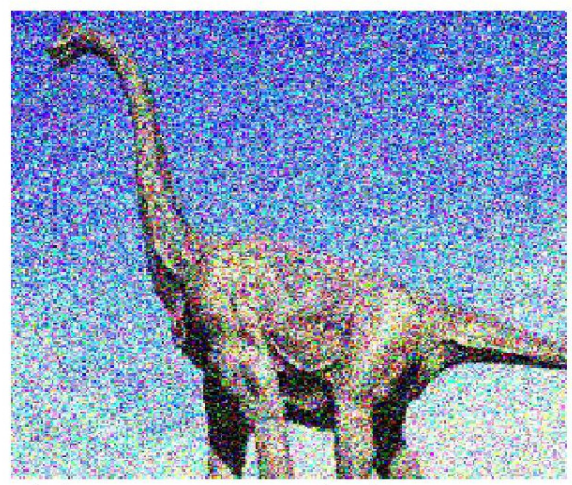

(g) Decrypted image using noisy cipher image

(Gaussian noise with mean $=0$ and variance $=0.15$ )

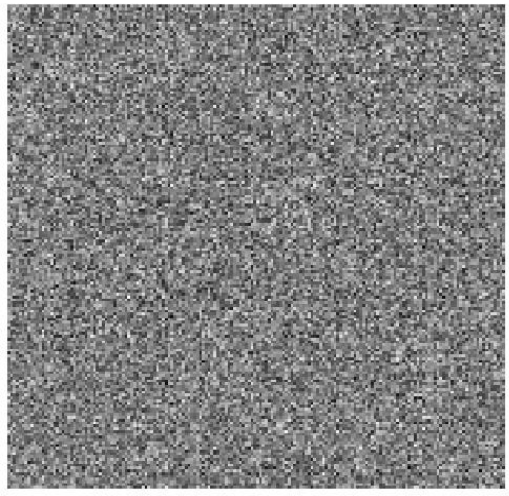

(c)The cipher image

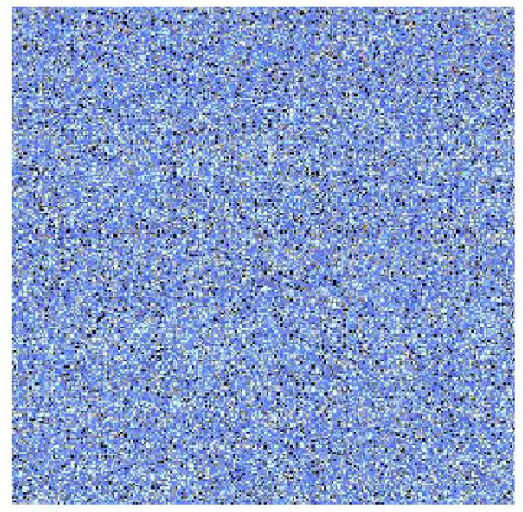

(f) Decrypted image using the wrong key (error in the key ratio $=1 / 1 \mathrm{E} 16$ )

Figure 6. The effects of encryption and decryption of dinosaur color image 
Through (7), we calculate the NPCR index for this color dinosaur image, it can get the value of NPCR as 0.9961 . The information entropy values of the original image and cipher image are 7.1376 and 7.9915 , respectively, it can be seen that the proposed algorithm works just as well for color image as for gray image. The NPCR and information entropy values for the cipher images of Lena, Cameraman and Dinosaur are summarized in Table 2.

\begin{tabular}{|l|l|l|}
\hline & NPCR & IE \\
\hline Lena $(256 \times 256)$ & 0.9960 & 7.9913 \\
\hline Cameraman $(256 \times 256)$ & 0.9961 & 7.9913 \\
\hline Dinosaur $256 \times 256)$ & 0.9961 & 7.9913 \\
\hline
\end{tabular}

Table 2. NPCR and information entropy of cipher Lena,Cameraman and Dinosaur images

\subsubsection{Sensitivity test of the Image Encryption Key for the Decryption}

The security of image encryption algorithms are determined by the encryption key. The more sensitive to the initial parameters value, the higher security is. In the experiment, if we change the image encryption key $x_{i}(i=$ $1,2, . ., 6)$ and step size $h$ by a very small value, such as change the initial value from $x_{1}(0)=0.1$ to $x_{1}(0)=0.1+1 \mathrm{E}^{-16}$, then the cipher image cannot be correctly decrypted. Based on this experiment, we can roughly estimate the size of the key space. Assuming that key values are within the range -1 to 1 and having seen that a change of $1 \mathrm{E}^{-16}$ in a single number of the key leads to unsuccessful decryption; then the number of possible keys can be approximated as $\left(2 / 1 \mathrm{E}^{-16}\right)^{6}=6.4 E^{97}$. Even a powerful computer that can try 1 trillion keys every second has no hope of guessing the key in any realistic amount of time.
4.2 Comparison with the State-of-the-art in Chaosbased Image Encryption

In the second round of experiments, we compared the proposed algorithm against three recently proposed algorithms for chaos-based image encryption [16,25,29]. We also compared with our own algorithm but using only $5 \mathrm{D}$ chaotic sequence, which to our knowledge is the highest-dimensional chaotic CNN before this work [6]. We used Lena of size $256 \times 256$ and Cameraman of size $512 \times$ 512 as test images. The result is shown in Table 3.

From the results it can be seen that our algorithm is better than the 5D CNN chaotic encryption algorithm and the Huang [16]algorithm for both the NPCR and the information entropy. Comparing to the Zhan algorithm [29] and the Wang algorithm [25], we see that the NPCR of our algorithm is equal to or better than these two algorithms. For the information entropy, the result for our algorithm is slightly less compared to the other two. Note that the Zhan algorithm [29] has very low NPCR value.

\subsubsection{Comparison of Robustness to Noisy Cipher Images}

When a cipher image is transmitted through a wireless channel, it is unavoidably affected by noise. In order to achieve good recovery of the original image, an algorithm must have certain ability to resist noise in the cipher image. In this experiment we verify the effectiveness of our algorithm for noisy cipher image, which contains added Gaussian noise with zero mean, and variance varying from 0.01 to 1 . We compared our algorithm with the same three algorithms as in the previous session. The result of decryption from noisy cipher images are shown in Figure 7. In order to more easily interpret the result, we also calculated the root mean square error (RMSE) between the decryption image and the original image at various

\begin{tabular}{|l|l|l|l|}
\hline \multirow{5}{*}{} & Algorithm & NPCR & IE \\
\cline { 2 - 4 } & CNN(5D)[6] & 0.9932 & 7.952 \\
\cline { 2 - 4 } & Huang et al.[16] & 0.9953 & 7.990 \\
\cline { 2 - 4 } & Zhan et al.[29] & 0.5974 & 7.996 \\
\cline { 2 - 4 } & Wang et al.[25] & 0.9961 & 7.996 \\
\cline { 2 - 4 } & Our algorithm & 0.9960 & 7.991 \\
\hline \multirow{5}{*}{ Cameraman $(256 \times 256)$} & CNN(5D)[6] & 0.9938 & 7.960 \\
\cline { 2 - 4 } & Huang et al.[16] & 0.9959 & 7.992 \\
\cline { 2 - 4 } & Zhan et al.[29] & 0.4987 & 7.996 \\
\cline { 2 - 4 } & Wang et al.[25] & 0.9961 & 7.997 \\
\cline { 2 - 4 } & Our algorithm & 0.9961 & 7.991 \\
\hline
\end{tabular}

Table 3. Comparing state-of-the-art chaotic encryption algorithms to our algorithm 
variances of the added Gaussian noise, shown in Figure 8 . It can be seen that all algorithms perform similarly when the variance of the added noise is low. At higher variance value starting from 0.1 onward, our algorithm clearly outperforms the other 3 in term of robustness to noisy cipher image.

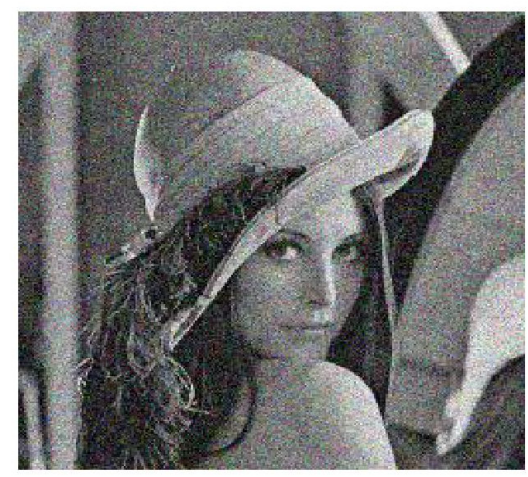

(a) $[16], \sigma^{2}=0.01$

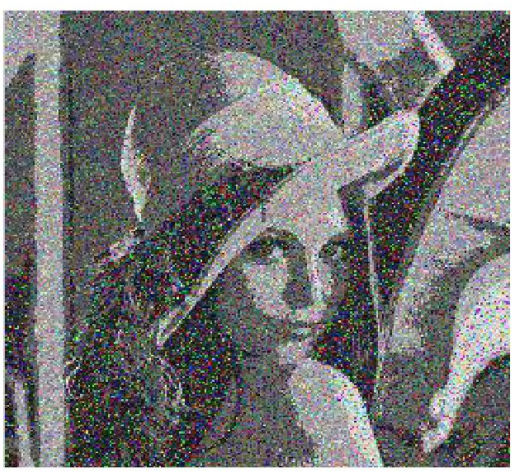

(d)[29], $\sigma^{2}=0.01$

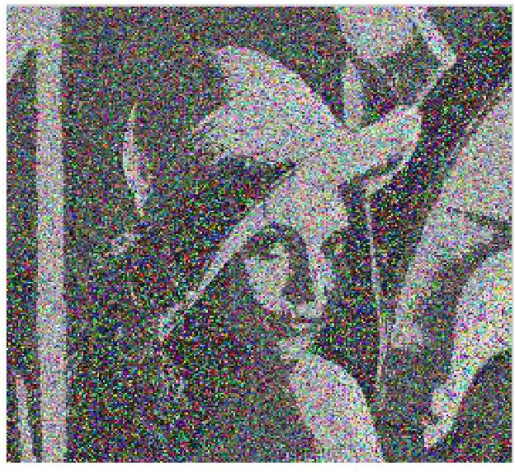

$(\mathrm{g})[25], \sigma^{2}=0.01$

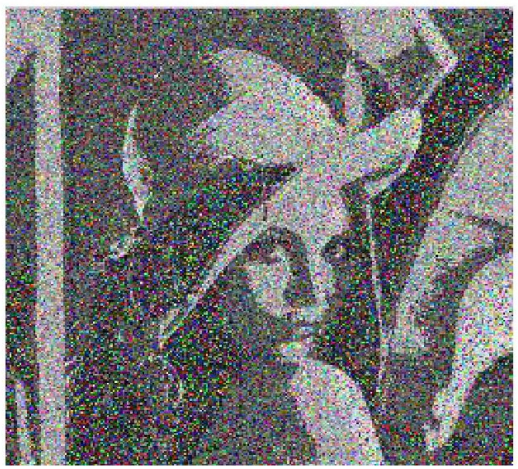

(j)Ours, $\sigma^{2}=0.01$



(b) $[16], \sigma^{2}=0.2$

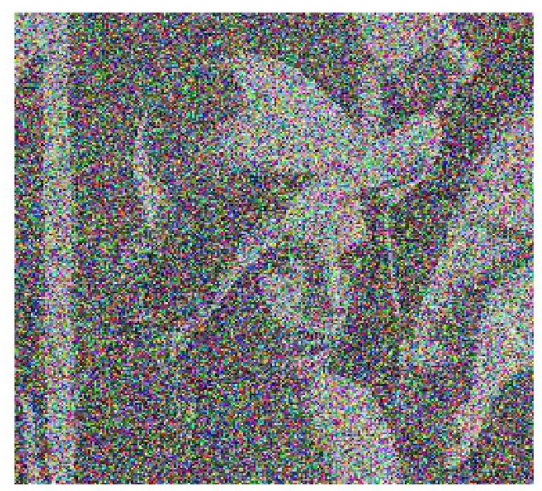

(e) $[29], \sigma^{2}=0.2$

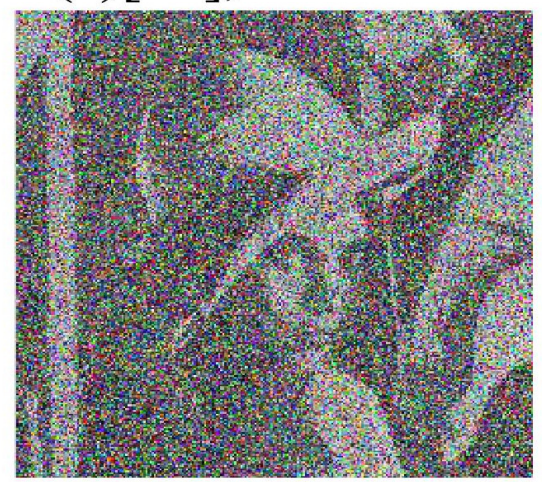

(h) $[25], \sigma^{2}=0.2$

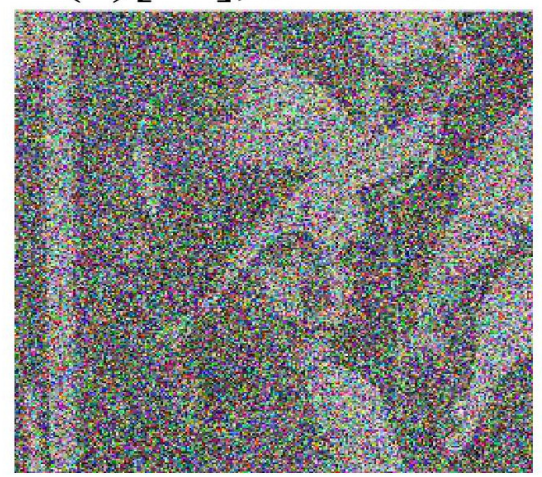

(k)Ours, $\sigma^{2}=0.2$

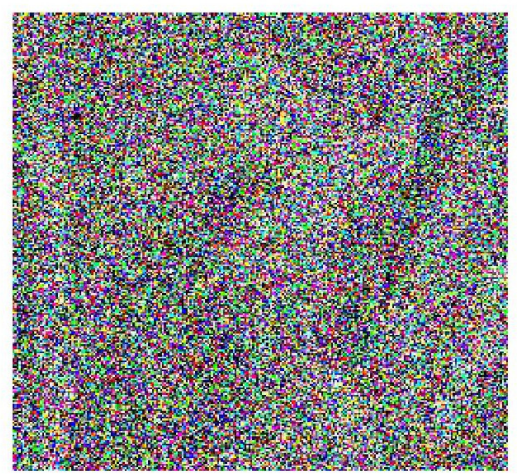

(c) $[16], \sigma^{2}=1$

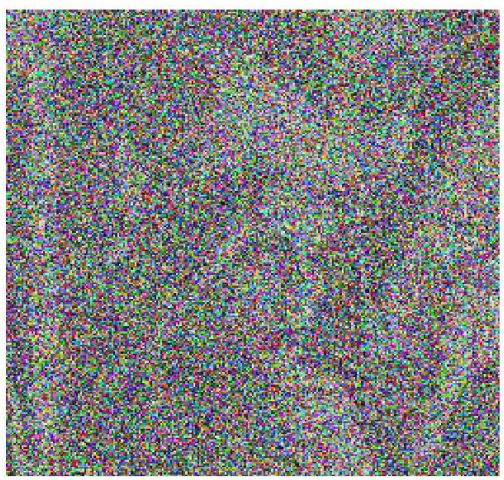

(f) $[29], \sigma^{2}=1$

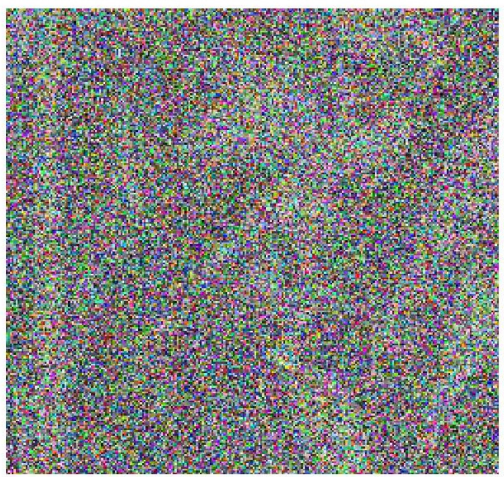

(i) $[25], \sigma^{2}=1$

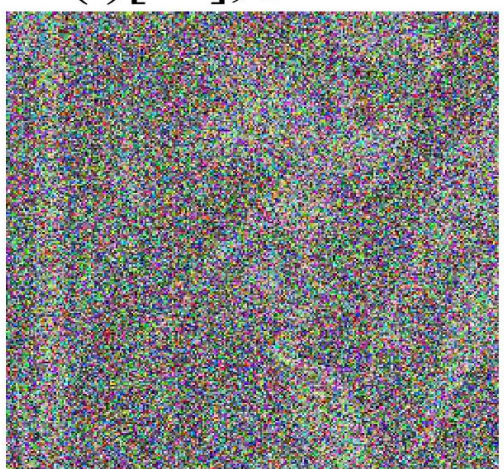

(1)Ours, $\sigma^{2}=1$

Figure 7. Decryption results when the cipher image is corrupted with noise. 


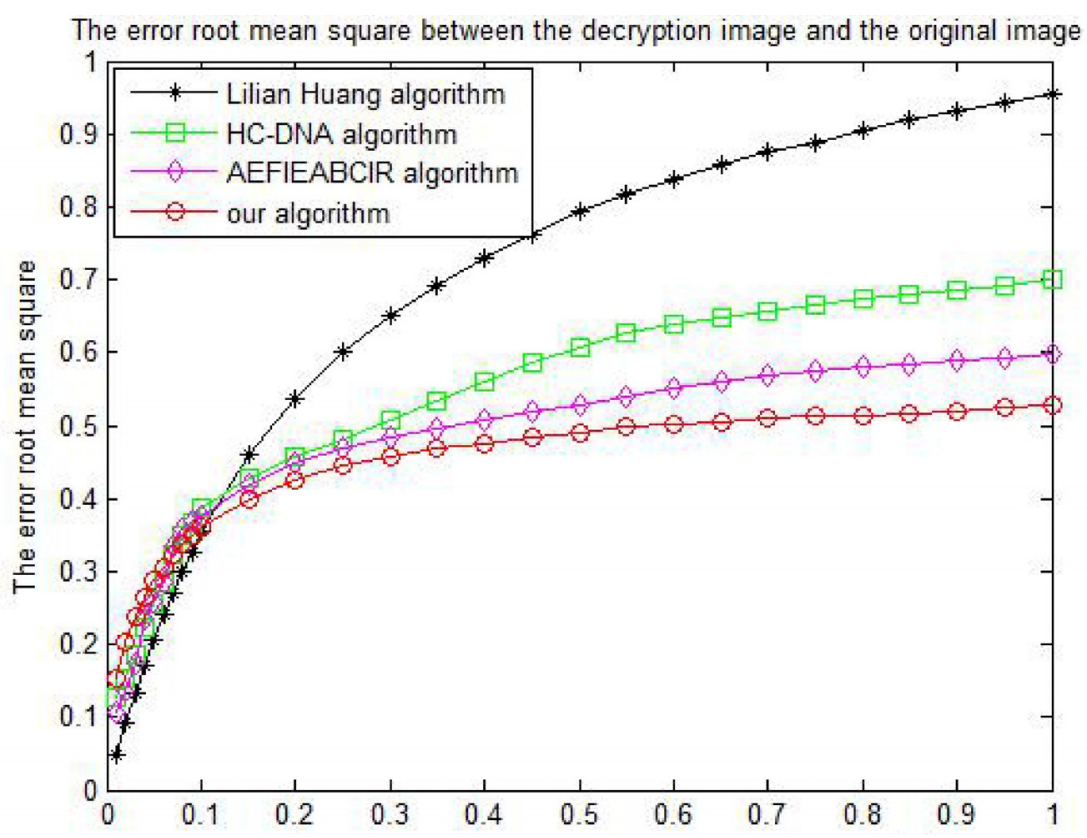

Figure 8. The RMSE between the decrypted image and the original image at various variance of the added Gaussian noise. For the plot legend: Lilian Huang is [16], HC-DNA is[29] and AEFIEABCIR is [25]

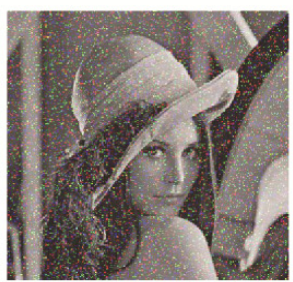

0.1143

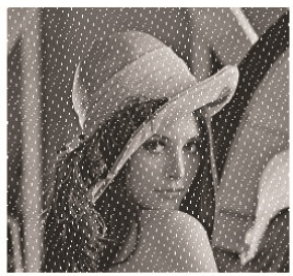

0.1337

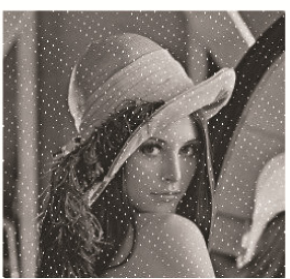

0.0445

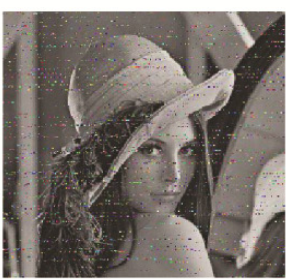

0.0377

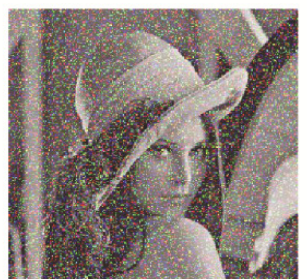

0.2285

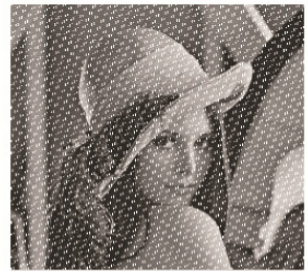

0.2077

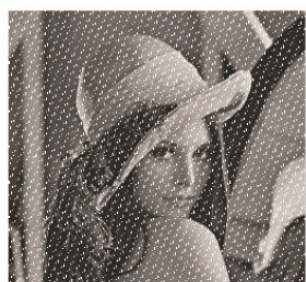

0.0887

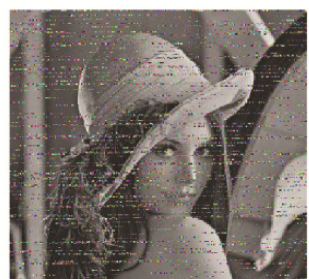

0.0758

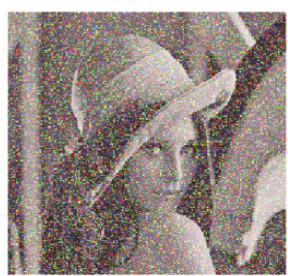

0.3419

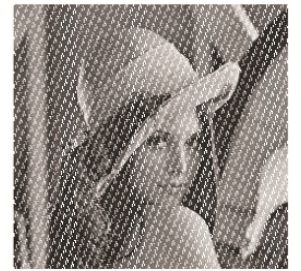

0.2856

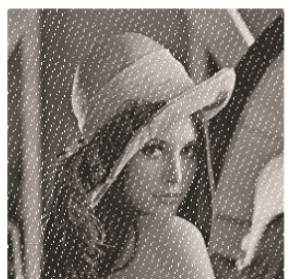

0.1270

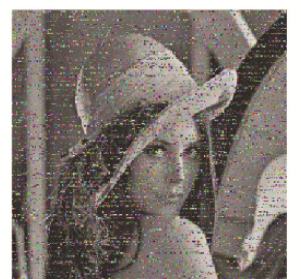

0.1144

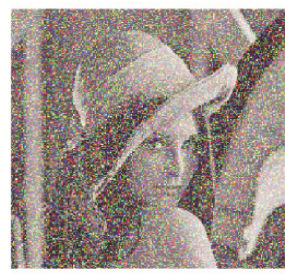

0.4568

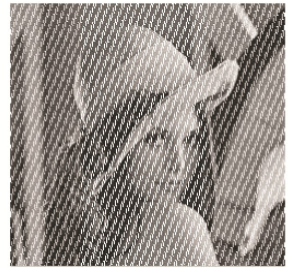

0.3378

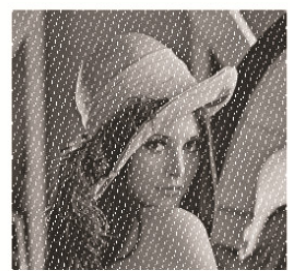

0.1648

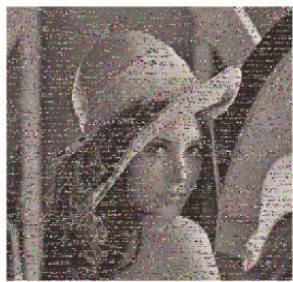

0.1525

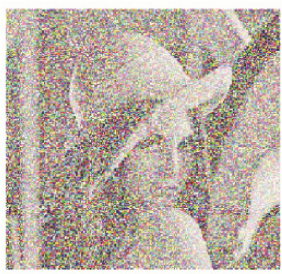

0.9106

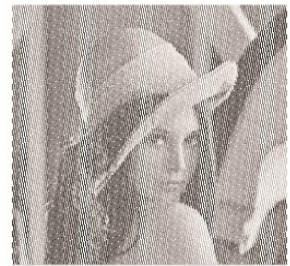

0.5560

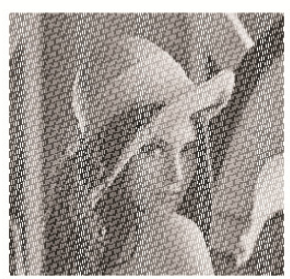

0.4832

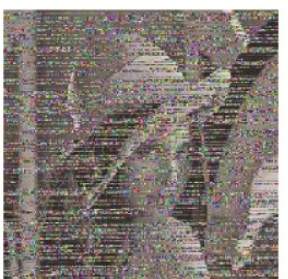

0.3038

Figure 9. Decrypted images from partially missing cipher images. The proportion of missing pixels are (from left column to right column) 6.25, 12.5, 18.75, 25, 50 percent respectively. (Row 1) Huang et al. [16]. (Row 2) Zhan et al.[29]. (Row 3) Wang et al.[25].

(Row 4) Our algorithm. The caption of each figure indicate the RMSE between the decrypted image and the original image 


\subsubsection{Comparison of Robustness to Missing Pixels in the Cipher Images}

Another possible scenario that can happen is part of the cipher image may be missing due to incomplete transmission or memory card corruption due to power failure when writing. In this experiment we compared how the algorithms perform when some part of the cipher image is replaced with white pixels. The result is shown in Figure 9. Each column of the figure corresponds to the decrypted images when the cipher image has 6.25, 12.5, 18.75, 25 and 50 percent of the pixels missing, respectively from left to right. The rows of the figure correspond to the result of 5D CNN, [16], [25], [29]and our algorithm, respectively. The number below each image indicate the RMSE value between the original image and decrypted image. It can be seen that our algorithm has the lowest RMSE value in every column, illustrating its superior robustness to partially missing cipher images.

\section{Conclusion}

In this paper, we proposed a $6 \mathrm{D}$ chaotic $\mathrm{CNN}$ based image encryption algorithm. Our algorithm achieved competitive performance when compared with recent stateof- the-art image encryption algorithm in terms of security, and outperformed them in terms of robustness to noisy cipher images and cipher images that are partially missing. The source of robustness comes from using the highdimensional chaos sequence directly as the basis of encryption together with operations that can be replicated exactly at the receiving end regardless of the corruption in the cipher image. By increasing the number of dimension of the chaotic sequence from 5 to 6 , the proposed algorithm was able to achieve security very close to the state-ofthe-art as measured by information entropy, while maintaining robustness.

Further work include testing the robustness against different kinds of non- Gaussian noise, fast implementation of the algorithm in hardware such as FPGA so that it can operate in real time/low power constraint, investigate the use of alternative binary representations for numbers, and finally improving the algorithm further by considering even higher dimensional chaotic CNN.

\section{Declaration}

\section{Funding}

This project was supported by the Doctoral Research Foundation of Southwest Forestry University (grant number 111802), it was also supported by the National Natural Science Foundation of China(grant number 31760181 and 61261013).

\section{Authors' Contributions}

The first author conducted the experiments, the second author drafted the manuscript and guided the experiments, the third and the fourth authors provided comments and feedback.

\section{Competing Interest}

The authors hereby declare that they have no competing interest.

\section{References}

[1] Assad OJSE, Chetto, M. (2017). Design and analysis of two stream ciphers based on chaotic coupling and multiplexing techniques. Multimedia Tools Applications 1 (6) 1-27.

[2] Behnia, S., Akhshani, A., Mahmodi, H., Akhavan, A. (2008). A novel algorithm for image encryption based on mixture of chaotic maps. Chaos, Solitons \& Fractals 35 (2) 408-419.

[3] Bhatnagar, G., Wu, Q. J. (2012). Selective image encryption based on pixels of interest and singular value decomposition. Digital signal processing 22 (4) 648-663.

[4] Bigdeli, N., Farid, Y., Afshar, K. (2012). A novel image encryption/decryption scheme based on chaotic neural networks. Engineering Application of Artificial Intelligence 25 (4) 753-765.

[5] Caragata, D., Tutanescu, I. (2014). On the security of a new image encryption scheme based on a chaotic function. Signal ImageVideo Processing 4 (8) 641-646.

[6] Chauhan, M., Prajapati, R. (2014). Image encryption using chaotic based artificial neural network. International Journal of Scientific and Engineering Research 5 (6) 351354.

[7] Chen, G., Mao, Y., Chui, C. K. (2004). A symmetric image encryption scheme based on $3 \mathrm{~d}$ chaotic cat maps. Chaos, Solitons \& Fractals 21 (3) 749-761.

[8] Chen, Jx., Zhu, Z. I., Fu, C., Yu, H., Zhang, Lb. (2015). A fast chaos-based image encryption scheme with a dynamic state variables selection mechanism. Communications in Nonlinear Science and Numerical Simulation 20 (3) 846-860.

[9] Chua, L. O., Yang, L. (1988).Cellular neural networks: theory. IEEE Transactions on Circuits and Systems 35 (10) 1257-1272.

[10] El Assad, S., Farajallah, M. (2016).A new chaosbased image encryption system. Signal Processing: Image Communication 4 (1)144-157.

[11] Fu, C., Chen, Jj., Zou, H., Meng, Wh., Zhan, Yf., Yu, Yw. (2012). A chaos-based digital image encryption scheme with an improved diffusion strategy. Optics Express 20 (3) 2363-2378.

[12] Gao, T., Chen, Z. (2008). A new image encryption algorithm based on hyper chaos. Physics Letters A 372 (4) 394-400.

[13] Guan, Z. H., Huang, F., Guan, W. (2005).Chaos-based image encryption algorithm. Physics Letters A 346 (1) 153-157.

[14] Guo, J. I. (2000). A new chaotic key-based design for 
image encryption and decryption. In: Circuits and Systems, 2000. Proceedings. ISCAS 2000 Geneva. The 2000 IEEE International Symposium on, IEEE, vol 4, 4952.

[15] Han, D., Min, L., Hao, L. (2016). A chaos robustness criterion for $2 \mathrm{~d}$ piecewise smooth map with applications in pseudorandom number generator and image encryption with avalanche effect. Mathematical Problems in Engineering 2016.1-14

[16] Huang, L., Shi, D., Gao, J. (2016). The design and its application in secure communication and image encryption of a new lorenz-like system with varying parameter. Mathematical Problems in Engineering 1-11

[17] Lin, M., Long, F., Guo, L. (2016). Grayscale image encryption based on latin square and cellular neural network. In: Control and Decision Conference (CCDC), 2016 Chinese, IEEE, 2787-2793.

[18] Pareek NK, Patidar V, Sud KK (2006).Image encryption using chaotic logistic map. Image and vision computing 24 (9) 926-934.

[19] Pareek, N. K., Patidar, V., Sud, K. K. (2013). Diffusion-substitution based gray image encryption scheme. Digital Signal Processing. 23 (3) 894-901

[20] Patidar, V., Pareek, N., Purohit, G., Sud, K. (2010). Modified substitution diffusion image cipher using chaotic standard and logistic maps. Communications in Nonlinear Science and Numerical Simulation 15(10):2755-2765

[21] Runhe, Q., Zhu, C., Liu, S. (2015). A chaos image encryption algorithm based on binary sequence and baker mapping. In: International Industrial Informatics and Computer Engineering Conference (IIICEC 2015), Xi'an,China

[22] Strogatz, S. H. (2014).Nonlinear dynamics and chaos: with applications to physics, biology, chemistry, and engineering. Westview press.

[23] Taha, M. A., Assad, S. E. (2017). Design and efficient implementation of a chaos-based stream cipher. Internet Technology and Secured Transactions 7 (2) 89-114.

[24] Telem, A. N. K., Segning, C. M., Kenne, G., Fotsin, H. B. (2014). A simple and robust gray image encryption scheme using chaotic logistic map and artificial neural network. Advances in Multimedia 2014 (12) 1-13

[25] Wang, X., Liu, C., Zhang, H. (2016). An effective and fast image encryption algorithm based on chaos and interweaving of ranks. Nonlinear Dynamics 84 (3)15951607. 26.

[26] Wang Y, Wong KW, Liao X, Xiang T, Chen G (2009).A chaos-based image encryption algorithm with variable control parameters. Chaos, Solitons \& Fractals 41 (4) 1773-1783.

[27] Wang, Y., liu, Z., Ma, J., He, H. (2016). A pseudorandom number generator based on piecewise logistic map. Nonlinear Dynamics 4 (83) 2373-2391.

[28] Wong, K. W., Kwok, B. S. H., Law, W. S. (2008). A fast image encryption scheme based on chaotic standard map. Physics Letters A 372 (15) 2645-2652.

[29] Zhan, K., Wei, D., Shi, J., Yu, J. (2017).Cross-utilizing hyperchaotic and dna sequences for image encryption. Journal of Electronic Imaging 26 (1) 013-021.

[30] Zhang, L., Liao, X., Wang, X. (2005). An image encryption approach based on chaotic maps. Chaos, Solitons \& Fractals 24 (3) 759-765.

[31] Zhu, Z. I., Zhang, W., Wong, Kw., Yu, H. (2011). A chaos-based symmetric image encryption scheme using a bit-level permutation. Information Sciences 181 (6) 11711186. 